\title{
LA RESILIENCIA DE SAN PEDRO TULTEPEC, LERMA FRENTE A LA ESCASEZ DE AGUA POTABLE DE LOS COMITÉS
}

\section{THE RESILIENCE OF SAN PEDRO TULTEPEC, LERMA AGAINST THE SHORTAGE OF DRINKING WATER OF THE COMMITTEES}

\author{
José Juan Méndez Ramírez ${ }^{1}$ \\ Carlos Alberto Mastachi Loza² \\ Belina Martínez Fajardo 3 \\ Martín Vázquez-Sánchez ${ }^{4}$
}

\section{Resumen}

El cambio climático que está experimentando el planeta en las últimas décadas, ha enfrentado al ser humano a situaciones extremas por fenómenos naturales y por las consecuencias derivadas del comportamiento que ha tenido sobre algunos recursos como la sobreexplotación del recurso agua, la excesiva contaminación y la ausencia de acciones dirigidas a su preservación, de ahí que es este trabajo se establece como objetivo situar el estado de la resiliencia en la localidad de San Pedro Tultepec con respecto a la escasez del agua potable. Para cumplir con el objetivo se parte del método cualitativo y observación de campo, aunado a ello la aplicación de la entrevista semiestructurada. Los resultados obtenidos destaca la resiliencia de la localidad por las actividades realizadas, además de la independencia de los comités por parte del municipio. Entre los hallazgos están los ejidatarios que controlan las Ciénegas de Chimaliapan. Aunque lo localidad es resiliente a la escasez del agua presenta procesos de urbanización acelerada y pérdida de las Ciénegas.

Palabras clave: Resiliencia; Agua; Escasez; Adaptación y Transformación.

\footnotetext{
${ }^{1}$ Dr. en Ciencias Sociales de tiempor completo, Facultad de Planeación Urbana y Regional, Universidad Autónoma del Estado de México (UAEMex),Toluca, México. E-mail: cidfino@yahoo.com

2 Dr. en Ciencias del Agua de tiempor completo, Facultad de Ingeniería, Centro Interamericano de Recurso del Agua (CIRA) Universidad Autónoma del Estado de México (UAEMex). E-mail: camastachil@uaemex.mx

3 Dra. en Desarrollo Internacional de tiempo completo, Facultad de Planeación Urbana y Regional, Universidad Autónoma del Estado de México (UAEMex), Toluca, México. E-mail: belygf@gmail.com

4 Estudiante de Doctorado en Urbanismo de la Facultad de Planeación Urbana y Regional. E-mail: martin_vz123@hotmail.com
} 


\section{Abstract}

The climatic change that the planet is experiencing in the last decades has confronted the human being to extreme situations due to natural phenomena and the consequences derived from the behavior that it has had over some resources such as over exploitation of the water resource, the excessive contamination and the absence of actions aimed at its preservation, hence, this work establishes the objective of situating the state of resilience in the town of San Pedro Tultepec with respect to the shortage of drinking water. To fulfill the objective, we start with the qualitative method and field observation, coupled with the application of the semistructured interview. The results obtained highlight the resilience of the locality due to the activities carried out, as well as the independence of the committees by the municipality. Among the findings are the ejidatarios that control the Ciénegas de Chimaliapan. Although the locality is resilient to water scarcity, it has accelerated urbanization processes and loss of the Ciénegas.

Keywords: Resilience; Water; Scarcity; Adaptation and Transformation 


\section{INTRODUCCIÓN}

El desarrollo del trabajo gira en torno a la resiliencia de la localidad de San Pedro Tultepec ante la escasez del agua, el cual se desarrolla en seis puntos: el primero aborda la problemática de ciudades en torno al agua, en el segundo se plantea la teoría y características de la resiliencia urbana bajo la cual se sustenta dicho trabajo. En el tercer apartado se describe la metodología y materiales a utilizar, en el cuarto punto se desarrollan los resultados de dicha investigación, posteriormente en el quinto punto se realiza la discusión en torno a los resultados y la teoría utilizada que se contrastó con los resultados obtenidos, finalmente se redactan las conclusiones.

\section{CONTEXTO DEL PROBLEMA}

El crecimiento demográfico y urbano de las ciudades trae consigo mayor demanda de infraestructura, alimentación, energía, vivienda y agua. La rápida urbanización es una de las mayores transformaciones sociales en la historia humana. Las ciudades han propiciado el agotamiento de los recursos y se enfrentan a nuevos riesgos causados por el cambio climático. También pueden ser los motores de la innovación. Por ejemplo, las ciudades de todo el mundo están adoptando medidas basado en la naturaleza para la adaptación y la resiliencia, tales como los techos verdes y la restauración de los humedales (MCPHEARSON et al., 2016).

El cambio climático ha propiciado problemas relacionados a la existencia o agotamiento de los recursos naturales, tal es el caso de la ciudad del Cabo, Sudáfrica, que enfrenta problemas de abastecimiento de agua. Presenta escasez del agua provocado por una prolongada sequía de tres años y por la falta de planeación del recurso que los pone una situación de riesgo de quedarse sin agua prevista como el "día cero" (SENGUPTA, 2018). Otro caso es el de Venecia, Italia ciudad en la que ha disminuido el nivel del agua por segundo año en el centro histórico, esta situación afecta en primer momento a la actividad turística en el uso de las góndolas (VANGUARDIA, 2018). De continuar esta tendencia los habitantes podrían enfrentar escases del recurso para el consumo humano.

Por ello, la planeación y desarrollo de la ciudad debe contemplar variables de diferentes ámbitos sociales y naturales orientados al bienestar de la población como: accesibilidad, áreas verdes, usos de suelo, la movilidad, espacios públicos, vialidades e infraestructura que permitan el funcionamiento de áreas urbanas o rurales, ello implica la ordenación y funcionamiento que 
requiere de diferentes disciplinas como: urbanistas, arquitectos, ingenieros, biólogos, sociólogos, abogados, economistas y ambientalistas.

En torno a este discurso se han perfeccionado nuevos enfoques de investigación que vinculan la relación armónica con los sistemas urbanos y el medio ambiente. Destaca la comunidad Disaster Risk Reduction (RRD) inicialmente ligada a la comprensión de la ingeniería, la capacidad de recuperación ecológica, y la capacidad de adaptación social (BÉNÉ et al., 2014). Enfocada al estudio del cambio climático y en el diseño de propuestas que mitiguen los desastres socio-naturales.

Para conseguirlo algunos gobiernos han buscado mejorar la capacidad de los sistemas urbanos y de infraestructura para hacer frente a los desastres (GIBBERD, 2014). Se ha puesto mucha a tención a los sistemas urbanos que dependen de recursos naturales, tales como el agua, enfocándose principalmente al manejo, distribución y consumo del líquido, a fin de corregir o dar respuesta al problema de escasez del agua, para afrontar dicho problema se recurren a diferentes acciones ya sean estructurales o no estructurales; la primera se relaciona con actividades de infraestructura como la construcción de pozos o la introducción y ampliación de la red de agua.

Las acciones no estructurales están relacionadas con las normas y acuerdos que se dan entre los comités ${ }^{5}$ de agua o el organismo operador encargado de administrar el agua municipal. Las afectaciones y la susceptibilidad de la disponibilidad del recurso ésta en función de la demanda, por lo tanto, la resiliencia urbana no solo comprende la parte física y social de la ciudad, sino que presenta una característica inherente, como son las ventajas naturales y oportunidades económicas (MACLUNE; TYLER; ALLAN, 2013). Por lo tanto, el agua condiciona cada contexto para el desarrollo urbano, como el caso de San Pedro Tultepec, municipio de Lerma para poder persistir. También, se ha considerado en la Agenda 2030 como uno de los objetivos para garantizar la disponibilidad del agua y su gestión (ONU, 2015)

A mayor crecimiento urbano de la localidad de San Pedro Tultepec, también incrementan las demandas sociales, dirigidas a satisfacer sus necesidades por medio del mejoramiento de la infraestructura de bienes y servicios. Uno de estos servicios es la demanda y disponibilidad de agua potable considerado por ROCKSTRÖM (et al., 2014) como una variable

\footnotetext{
${ }^{5}$ La conformación de esta figura corresponde a un vacío jurídico que da legalidad a organizaciones comunitarias que realizan una administración y operación eficiente en el manejo de sus recursos (LÓPEZVILLAMAR; MARTÍNEZ-SALDAÑA; PALERM-VIQUEIRA, 2013).
} 
de control para el soporte de las áreas urbanas. Para evaluarlo se caracterizan tres aspectos que fomentan la resiliencia urbana: diversidad, redundancia y modular.

\section{ACERCAMIENTO TEÓRICO DE LA RESILIENCIA URBANA}

La resiliencia se asocia con la vulnerabilidad y ésta llegó a ser desarrollado por Engels en 1945, cuando se refirió a la vulnerabilidad de la clase obrera. Fue propuesta de manera explícita desde la perspectiva de los desastres y se maneja desde la escuela ecologista a mediados del siglo XX (CARDONA, 2005). Dentro de este marco las condiciones de vulnerabilidad determinan el estado de resiliencia, estos dos términos son inherentes, ya que existen múltiples elementos que condicionan el desarrollo de la población y, al igual que la ciudad post-industrial, la ciudad sostenible y la ciudad resiliente son necesidades particulares que caracterizan a una fase de la sociedad (CAPUTO et al., 2015).

La resiliencia urbana parte del contexto teórico de la Social-ecological resilience, se orienta a esta corriente teórica por las acciones dinámicas que busca la estabilidad desde la interrelación de múltiples escalas para adaptarse y transformarse continuamente y todavía permanecer dentro de los umbrales críticos (FOLKE et al., 2010). Por resiliencia urbana se entiende como: "la capacidad de una ciudad para absorber los efectos de las conmociones y tensiones plausibles, recuperarse rápidamente de ellas y mantener la continuidad de los servicios" (ONU-HABITAT, 2017)

La noción de resiliencia ha sido empleada por distintas disciplinas, entre las que se encuentran la planeación y el urbanismo, quienes han incorporado algunos principios de ésta en el diseño de los instrumentos de la planeación, dirigidos a dar respuesta a los escenarios de vulnerabilidad y riesgo que son cada vez más constantes en los últimos años.

El urbanismo propone desarrollar investigaciones, haciendo uso de la resiliencia destacando procesos de mejora, medidas eficientes mediante la retroalimentación y la planeación de las ciudades con la integración de los recursos naturales a corto, mediano y largo plazo. Para el desarrollo de este trabajo se adopta la propuesta del enfoque ecológico, específicamente lo relacionado con dos propiedades: la adaptación y transformación. (HOLLING, 1973) y de las características: diversidad, redundancia y modular (TKF, 2015) 


\section{CARACTERÍSTICAS DE LA RESILIENCIA URBANA}

Al hacer uso de la resiliencia urbana es posible determinar cómo intervenir en la ciudad con el propósito de aumentar la capacidad de adaptación y transformación, son el complemento para el desarrollo urbano llevada a cabo mediante la planeación y la realización de acciones estratégicas. Las características que se retoman en esta investigación son las que determinarán por medio de las acciones colectivas la resiliencia de los comités de agua, estas características son: la diversidad, redundancia y modular.

La diversidad hace referencia a los numerosos componentes del sistema urbano que tienen una amplia gama de respuestas al cambio, por lo tanto, es poco probable que fracasen. Por ejemplo, una ciudad con una base económica diversa es menos vulnerable a la agitación económica que una que depende de una sola actividad industrial. En la gobernanza y la toma de decisiones, un proceso de colaboración que incorpora una variedad de actores y perspectivas es probable que produzca mejores resultados. La redundancia tiene múltiples formas de realizar funciones básicas, de modo que el fallo de un componente no provoca que todo el sistema se bloquee. Lo modular en la resiliencia urbana se refiere a las unidades individuales que conservan cierta autosuficiencia cuando se desconectan de redes más grandes se sentirán mejor en tiempos de cambio (TKF, 2015).

Estas características se relacionan con los componentes urbanos como la infraestructura, usos de suelo y la estructura demográfica (SHARIFI; YAMAGATA, 2014). La ciudad no es ajena a elementos externos, principalmente por la dependencia que tiene con el medio natural, del cual extrae los recursos necesarios para su funcionamiento, como el agua. La dependencia de áreas urbanas con el recurso agua ésta en función de la duración de las fuentes de abastecimiento e infraestructura o por la misma existencia del recurso determinada por las condiciones físico-naturales.

\section{CARACTERIZACIÓN DE SAN PEDRO TULTEPEC}

El municipio de Lerma se encuentra localizado entre cadenas montañosas, sierras, valles, cerros y hundimientos y ocupa el Monte de Las Cruces donde se asienta principalmente la población (GEM, 2013), tiene una superficie de 23,258.76 hectáreas distribuido en diferentes usos: agrícola $58.62 \%$, zona urbana $11.85 \%$, bosque $22.7 \%$, pastizal $4.63 \%$, el uso industrial con 
el $3.41 \%$ y los cuerpos de agua cubren el $3.13 \%$ de la superficie municipal (GEM, 2009). Cuenta con 78 localidades, entre las que se encuentra San Pedro Tultepec.

La localidad de San Pedro Tultepec presentó un incremento de población a partir de 1940, debido a la construcción del Acueducto que hoy se conoce como el "Alto Lerma"; la cuenca hidrológica del "Alto Lerma" representó una solución para el abastecimiento de agua de la ciudad de México, este acueducto es considerado como uno de los proyectos más importantes de los últimos cincuenta años, por la cantidad de agua que se extrae y se dirige a la ciudad, por el grado de explotación del recurso es considerado como uno de los que más ha perjudicado la ecología del Valle. (GEM, 2016),

San Pedro Tultepec, destaca la combinación de actividades agrícolas, ganaderas y lacustres (CAMACHO, 2007), entre la actividad lacustre destacaba la pesca, la caza, y la recolección, con fines de autoconsumo, productos que proveía la laguna (GONZÁLEZ et al., 2011). Recientemente la actividad lacustre ya no representa prioridad para la subsistencia de la comunidad ya que se encuentra en el proceso de desecación la laguna de Chimaliapan, representa también una fuente de abastecimiento de agua, las actividades industriales y el incremento de descargas de aguas residuales domésticas ha hecho que se vea afectada la disponibilidad del agua, propiciando la escasez y el cambio de vida lacustre.

Figura 1. Ubicación de la localidad de San Pedro Tultepec, Lerma

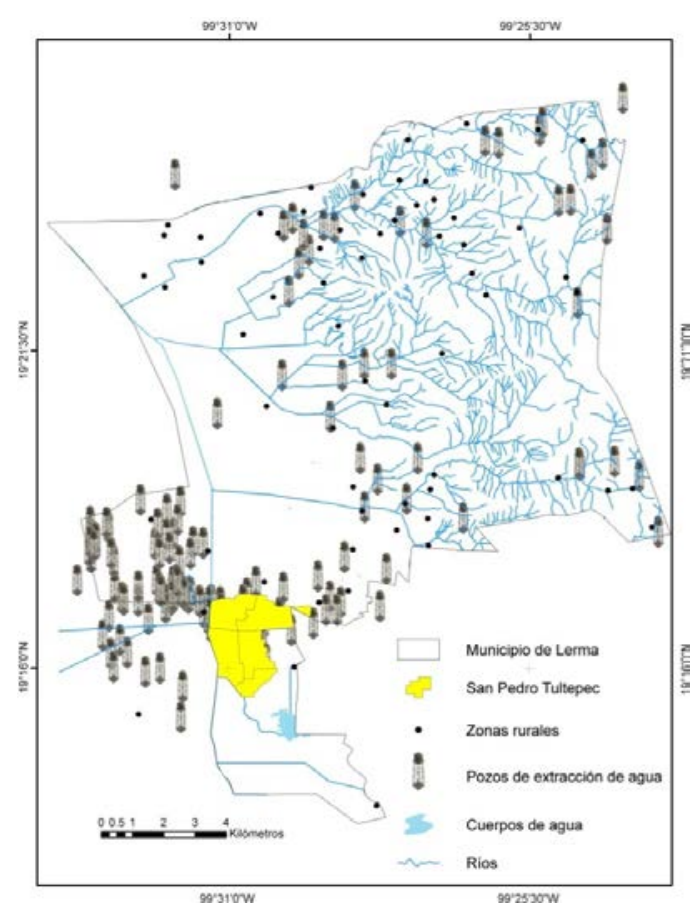

Fuente: elaboración propia con base en INEGI, 2010 
Con base a la observación y recorridos de campo en San Pedro Tultepec la disponibilidad y demanda del agua potable presente afectaciones por el aumento de la población, contaminación, pérdida de cubierta vegetal y la creciente expansión urbana en torno a la Ciénega Chimaliapan. Se suma también la escasa gestión, distribución desigual, concentración del servicio, las descargas de aguas domésticas e industriales sin previo tratamiento y crecimiento desordenado.

Las afectaciones a este cuerpo de agua han provocado la escasez del agua, situación que se conjuga con la falta de mantenimiento a la infraestructura de agua, con la que se satisface la demanda de este recurso por parte de la población, la falta de mantenimiento no permite que se disponga del servicio de manera constante y se presenten problemas de distribución. El encargado del agua en Lerma menciona que el problema se debe a la antigüedad de las tuberías, que va de 30 a 40 años, esta situación se agudiza por la incapacidad que muestran las tuberías para dotar del servicio y en temporadas de estiaje (Febrero-Mayo) el agua se distribuye por medio de pipas a las comunidades afectadas y a pesar de contar con comités autónomos que controlan el agua (hasta 4 en comunidades) presentan problemas de escasez como es el caso de San Pedro Tultepec.

Aún con los decretos de 1949 y el último de 1965 que protege el acuífero del Valle de Toluca, no permitió la recuperación del agua de la localidad de San Pedo Tultepec, a pesar de las medidas tomadas no se ha logrado reducir el problema por la constante extracción principalmente doméstico, industrial y agrícola, se suman también la demanda para la Ciudad de México. Esta situación ha orillado a la población de esta localidad adoptar medidas con las que consigan adaptarse a la nueva realidad.

\section{MÉTODOS Y MATERIALES}

Para medir la adaptación y transformación se hace uso del método cualitativo y para recabar información se base en la observación no participativa, notas de campo y las fuentes de información en textos, sitios web y fotografías. Para medir las categorías de análisis de la resiliencia urbana se retoman también las características en la que se ubicarán las actividades realizadas que fomentan la resiliencia urbana. Las actividades realizadas se obtendrán a través de una entrevista semiestructurada aplicada a dos comités de agua potable, para su aplicación se recurrió a la técnica bola de nieve. El muestreo por bola de nieve es una técnica de muestreo no probabilística en la que los individuos seleccionados para ser estudiados reclutan a nuevos 
participantes entre sus conocidos. Es un proceso económico y sencillo y requiere poca planificación y pocos recursos humanos: los propios sujetos entrevistados hacen de mano de obra (OCHOA, 2015). La entrevista se realizó conforme a la tabla 1.

Las dos categorías de análisis a evaluar son la adaptación y transformación, la primera retoma el aspecto ambiental, social y la parte económica que define la accesibilidad o alcance de los servicios básicos como el agua potable. Para la transformación urbana se enfoca principalmente a políticas y estrategias de planeación en la dotación del agua realizados por los comités.

Tabla 1. Contraste entre resiliencia urbana con la entrevista

\begin{tabular}{|c|c|}
\hline Característica & Pregunta \\
\hline Diversidad & $\begin{array}{l}\text { 1. ¿Usted toma decisiones entre grupos para distribuir el agua?7 } \\
\text { 2. ¿Existe una coordinación de los comités con el municipio para distribuir } \\
\text { el agua? } \\
\text { 3. ¿Qué porcentaje del presupuesto del comité se destina a obras del agua } \\
\text { e infraestructura? }\end{array}$ \\
\hline Redundante & $\begin{array}{l}\text { 4. ¿Usted se relaciona con otros comités para compartir experiencias de } \\
\text { distribución de agua potable? } \\
5 . \quad \text { ¿Los líderes del grupo promueven el cambio mediante reglas o normas } \\
\text { para una mejor distribución del agua? }\end{array}$ \\
\hline Modular & $\begin{array}{l}\text { 6. ¿Cuenta con fuentes alternas de agua potable (pozos, manantiales o } \\
\text { red de agua)? } \\
7 . \\
\text { ¿Tiene proyectos para cuidar el agua? }\end{array}$ \\
\hline
\end{tabular}
Fuente: elaboración propia

La tabla uno presenta diferentes actividades a realizar como estrategias para que los comités sean resilientes, se realizaron siete cuestionamientos de acuerdo a las condiciones de los dos comités en disponibilidad y disposición. Los comités, controlan el agua, para su distribución hacia ciertos sectores de la población, están enfocados principalmente en áreas rurales con menor población en diferentes secciones o barrios no en su totalidad del territorio municipal y los Organismo Público Descentralizado para la Prestación de los Servicios de Agua Potable, Alcantarillado y Saneamiento (OPDAPAS) administran principalmente a conjuntos urbanos y de zonas industriales que les concesionan el agua. 


\section{MEDIDAS DE RESPUESTA PARA AUMENTAR LA RESILIENCIA EN SAN PEDRO TULTEPEC}

Las medidas para aumentar la resiliencia de la localidad de San Pedro Tultepec, están relacionados con tres características. La primera es la diversidad, se refiere a la colaboración, decisiones y una serie de normas para mantener el agua así como la participación de diversos actores, la segunda, la redundancia se refiera a las alternativas u opciones de suministro de agua para no afectar las funciones urbanas y la tercera, modular se relaciona con la autosuficiencia y autonomía de modo que cualquier función que esté en crisis no sea afectado (TKF, 2015)

\section{Diversidad}

La diversidad de la localidad de San Pedro Tultepec se presentan en los comités autónomos, cada uno realiza funciones básicas de suministrar el agua a los usuarios, pero el fallo de cualquiera de ellos provoca que el servicio se interrumpa, las mayores afectaciones en la interrupción del servicio se presentan en zonas urbanas en las que el gobierno municipal controla este recurso, por su parte, las áreas rurales no son afectadas drásticamente por la interdependencia y autonomía con que cuentan los comités autónomos.

Un tercer actor que participa de manera significativa en el control del agua de la localidad de San Pedro Tultepec, son los ejidatarios de la Ciénega de Chicognahuapan, éstos influyen de manera indirecta en el cuidado y conservación del agua, esto por la ausencia de coordinación con los diversos comités autónomos, así como con las autoridades del gobierno municipal y de la ciudad de México. Esta situación impide a alguno de los actores referidos responsabilizarse de este recurso, para preservarlo y controlar su explotación.

La reforma al artículo 27 constitucional, vino a complejizar, aun más, la situación del recurso agua de la Ciénega, con dicha reforma se abrió la posibilidad de privatizar este recurso, lo que provocó problemas de disponibilidad, principalmente por la sobre explotación y extracción del agua. Cabe señalar que el deterioro de este cuerpo de agua, así como de su sobre explotación por los actores referidos, se debe a la ausencia de una reglamentación técnica que regule el acceso, la explotación y sancione a quienes no se apeguen a la normatividad. Al mismo tiempo, la falta de reconocimiento legal de los Comités Autónomos del municipio limita regular 
desde la ley el actuar de estos ya que, solo son reconocidos los inversionistas de la iniciativa privada mediante las concesiones y los organismos operadores del agua municipal.

\section{Redundancia}

La adaptación de la población por medio de la redundancia en la localidad de San Pedro Tultepec y la modificación de un modo de vida en torno al agua han permitido que sean resilientes, sin embargo la disponibilidad del agua natural subterránea y superficial está disminuyendo y como fuente principal afectará al sistema social. Ante la escasez del agua la población ha tomado medidas de adaptación como tomas clandestinas de agua y el uso común de posos artesanales, figura 2. Acciones que dependen de la disponibilidad del recurso, de ahí que estas medidas serán poco útiles a medida que el agua sea más escasa.

Figura 2. Medidas de adaptación

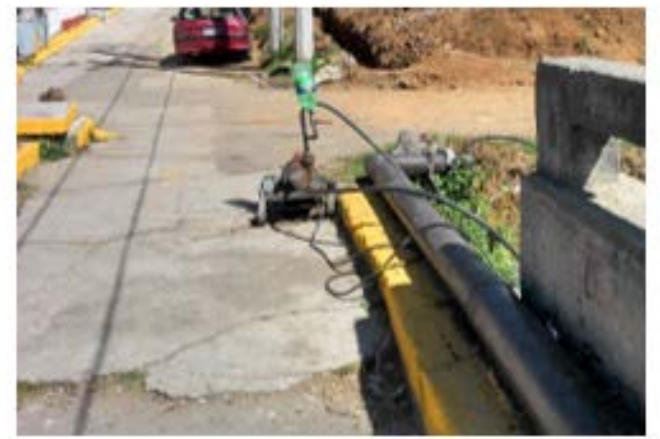

Centro de Lerma

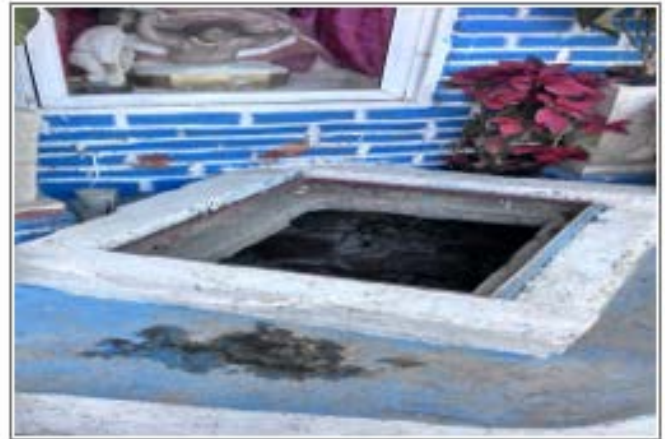

San Pedro Tultepec

Fuente: Fotografías tomadas en campo, 21/01/2017

El proceso de adaptación de la población de la localidad de San Pedro Tultepec es poco significativo, por ser un espacio receptor de flujos migratorios que producen u crecimiento urbano demográfico acelerado, son nuevos residentes que no sienten conexión con el medio natural del municipio y provocan contaminación de los cuerpos de agua y mantos freáticos, teniendo como consecuencia una menor disponibilidad del recurso.

El crecimiento urbano, motivado principalmente por la construcción de vivienda, presenta escasa planificación en el ordenamiento territorial, con ello, se han perdido espacios verdes que no sólo contienen flora que mantiene el equilibrio del clima, sino también limita las 
recargas acuíferas, en condiciones de escasez del recurso, esta población no cuenta con cuenta con otras fuentes alternas de agua, sólo cuentan con tanques cisternas que fueron construidos en 1966, pero que no se utilizan (ver figura 3). A esto se le suma el problema que representa la falta de mantenimiento de la infraestructura hidráulica por parte de los comités autónomas a cargo del recurso, ni por ningún nivel de gobierno.

Figura 3. San Pedro Tultepec, tanque cisterna

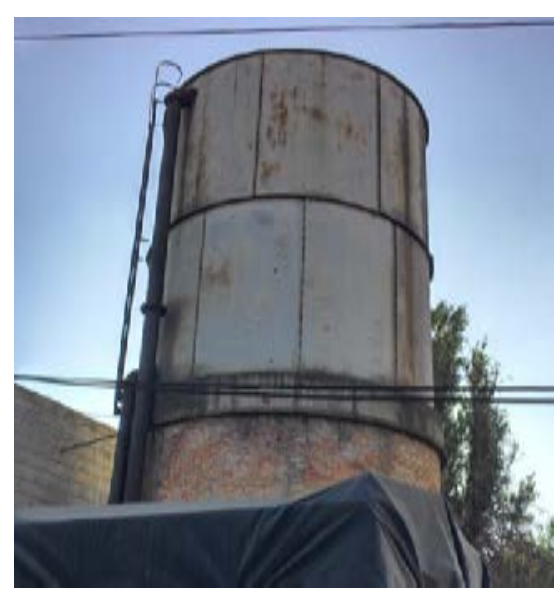

Fuente: Fotografías tomadas en campo, 21/01/2017

\section{Modular}

Las medidas tomadas para mantener el agua potable por el comité de San Pedro Tultepec se centran principalmente en la construcción de nuevos pozos y en el mantenimiento de la red de distribución. En cuanto a las medidas ambientales, los comités, gobierno municipal e iniciativa privada no han llevado a cabo acciones que impacten significativamente en la disponibilidad del agua. Por el contrario, el proceso de crecimiento urbano de la localidad de San Pedro Tultepec invade las Ciénegas reduce la vegetación y crea las condiciones para que se lleve a cabo un ecocidio en esta localidad. 
Figura 4. Proceso de ocupación de la Ciénega de Chimaliapan

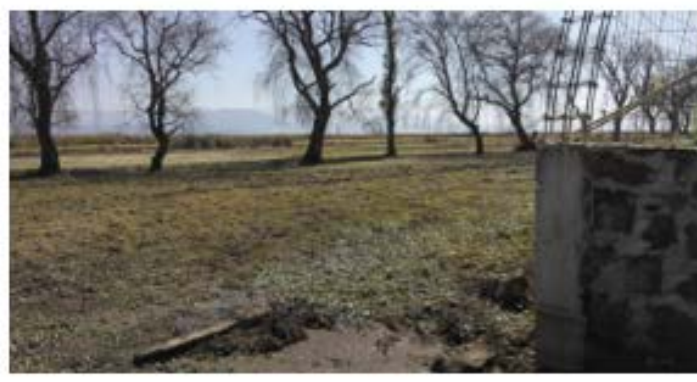

Construcciones de Vivienda

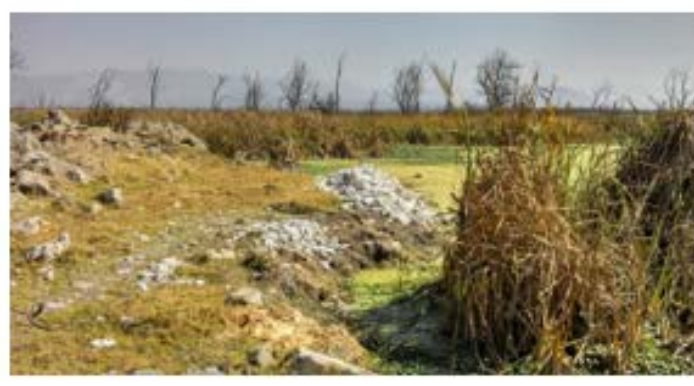

Desecho de construcciones

Fuente: Fotografías tomadas en campo, Ciénega de Chimaliapan, Lerma, 21-01-2017.

El municipio de Lerma cuenta con el Ordenamiento Ecológico pero, no se toma en cuenta para proteger las zonas frágiles de la Ciénega de Chimaliapan. A manera de resumen, en la figura 5 se hace una relación de las medidas de respuesta con las características de la resiliencia, esto se determinó con base a la entrevista realizada en la localidad de San Pedro Tultepec el cual es resiliente ante la escasez del agua potable, ya que realizan actividades de adaptación y transformación, aunque presenta un proceso de crecimiento e invasión de la Ciénega de Chimaliapan aún mantiene la capacidad el comité para disponer del agua.

Figura 5. Factores y actores que determinan la resiliencia en San Pedro Tultepec, 2010-2017

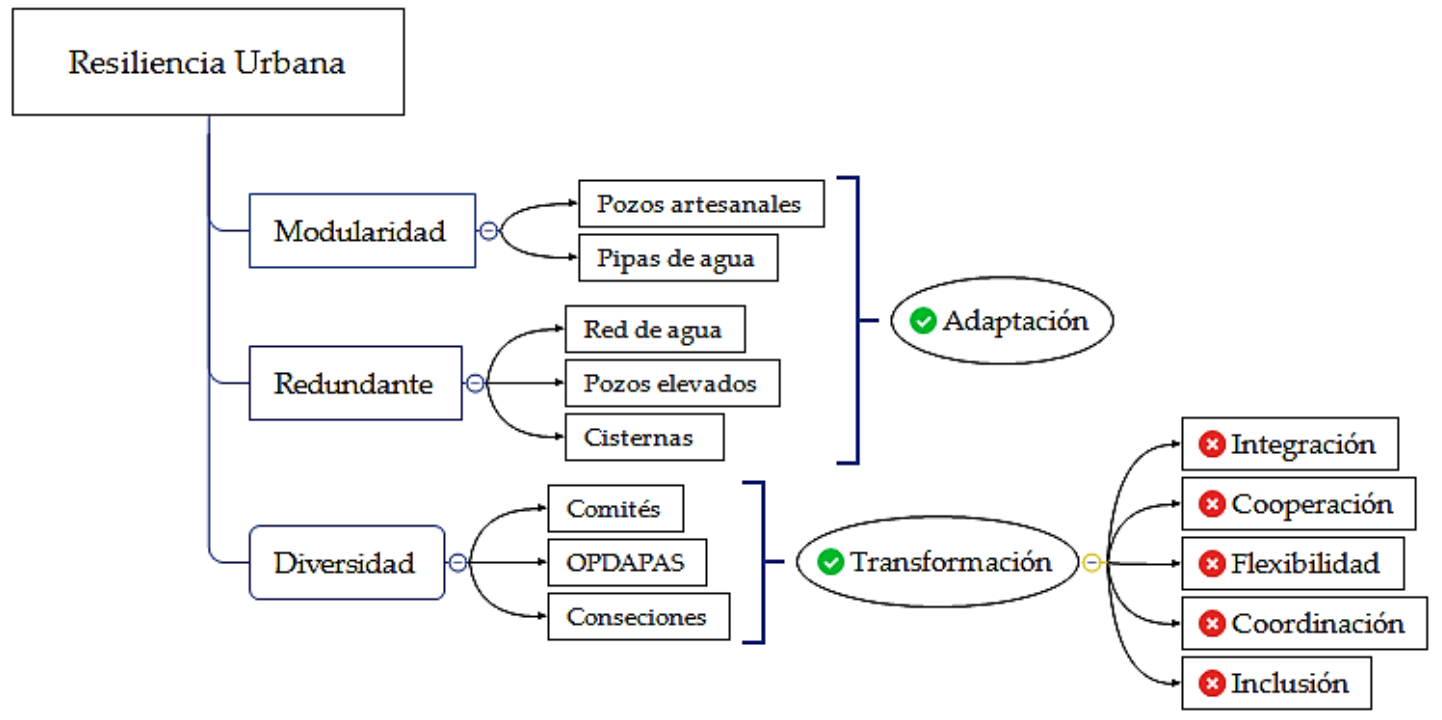

Fuente: elaboración propia 
Los factores que determinan la adaptación de San Pedro Tultepec están dados por la modularidad y redundancia, aunado a la diversidad los actores como los comités, OPDAPAS municipal e iniciativa privada que controlan el agua a través de concesiones o acuerdos, políticas aislados y parciales sin planeación a futuro, estos actores por la independencia que tienen presentan diferentes respuestas frente a la escasez del agua potable, por ende mantienen un nivel estable en cuanto a la disposición del agua en los municipios que les permite transformarse.

Respaldados por las reformas que experimentaron los artículos 27 y 115 constitucional, la iniciativa privada empieza a tener más atribuciones e injerencias en el control del agua, caso contrario al de los comités autónomos de las localidades del municipio, éstos no presentan algún respaldo jurídico que los reconozca como organismo operador, solo son respaldados por usos y costumbres en la administración del agua, sin embargo, es el actor que presenta mayor resiliencia por la autonomía y autosuficiencia.

Tanto los comités como los OPDAPAS trabajan de manera independiente, por lo que no se presenta una interacción en conjunto y una visión a futuro para mantener el agua en la localidad de San Pedro Tultepec. Además de que las normas que permiten el control del agua no fomentan la integración, cooperación, coordinación e inclusión social, por lo que se presentan conflictos sociales en quiénes deben administrar el agua, aunque se presenta este tipo de conflictos se ha mantenido la resiliencia de la localidad y refuerza la independencia e interdependencia de los actores para mantener la capacidad de adaptación y transformación, en la figura 6 se muestra de manera gráfica la dinámica de la resiliencia de San Pedro Tultepec.

\section{Discusión}

En la figura 6 se muestran los factores de vulnerabilidad, el umbral y la estabilidad que favorecen la resiliencia de los comités autónomos del agua potable en la localidad de San pedro Tultepec, haciendo uso de los principios de la transformación y adaptación y cómo los comités orientan las situaciones y procesos. 
Figura 6. Dinámica de la resiliencia en San Pedro Tultepec, Lerma

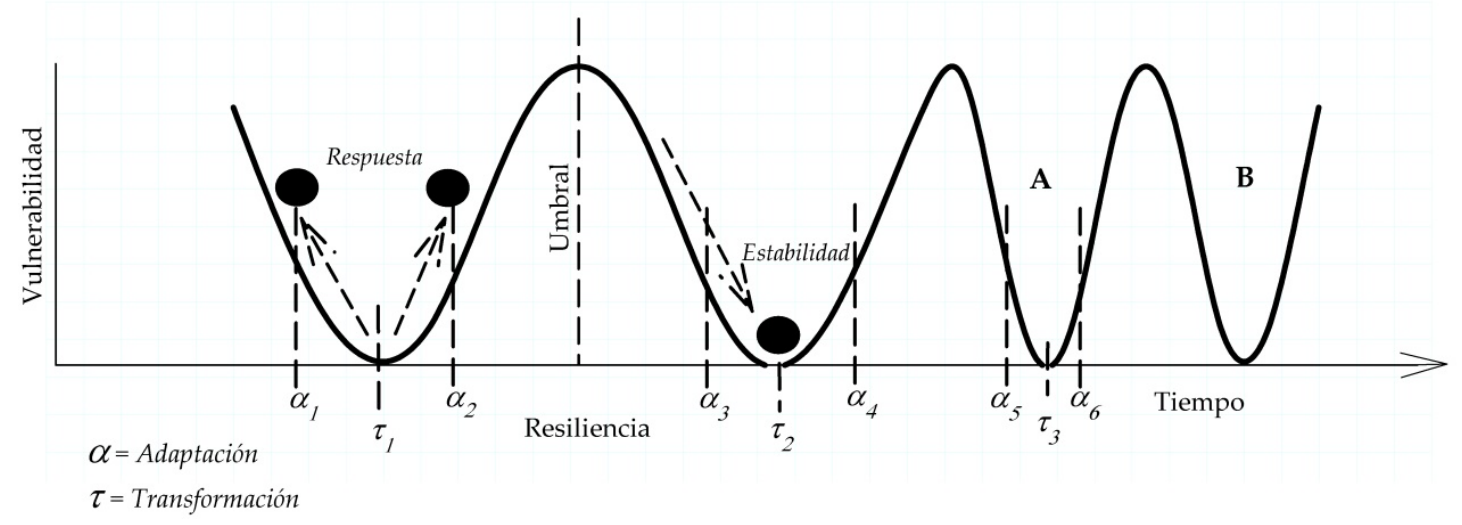

Fuente: elaboración propia con base en LIAO (2012).

La localidad de San Pedro Tultepec se ubica en el proceso de respuesta, donde $\alpha_{1}$ y $\alpha_{2}$ representan las oscilaciones durante la etapa de escasez del agua, encontrándose en el proceso de la adaptación y $\tau_{1}$ la estabilidad cuando la crisis de escasez del agua se supera y se transforma. Cuando se inicia el proceso de adaptación al mismo tiempo se presenta la transformación y dada la dinámica de la demanda y disponibilidad del agua, se comienza el proceso hacia el umbral para alcanzar la transformación.

Durante el proceso de adaptación se presentan oscilaciones como respuesta al cambio, a medida que trascurre el tiempo la presión que ejerce la escasez del agua conlleva a una situación crítica, según HOLLING (1973) obliga a cruzar el régimen o umbral. La adaptación será más rápido a medida que pasa el tiempo, puesto que se llevó a cabo un proceso de retroalimentación previa, y las medidas de respuesta serán menores realizadas a corto plazo, esto se expresa de la siguiente manera.

$$
\text { Adaptación }=\alpha_{1}=\alpha_{2}<\alpha_{3}=\alpha_{4}<\alpha_{5}=\alpha_{6} \quad 1
$$

Asimismo, al cruzar el umbral se estará situando en la estabilidad entre demanda y disponibilidad del agua, es la etapa donde las medidas estructurales y no estructurales de cada municipio favorecen la transformación con un proceso prolongado de respuesta, en el trascurso del tiempo la transformación será más rápido por las acciones previas implementadas, esto se expresa de la siguiente manera. 


\section{Transformación $=\tau_{1}<\tau_{2}<\tau_{3}$}

Las fases de respuesta serán eficaces y eficientes a medida que se implementen durante la crisis a lo largo del tiempo, logrando así la estabilidad, por lo que el punto A y B de la figura 2 representa una transición rápida de adaptación y transformación a corto plazo, favoreciendo el aumento de la resiliencia urbana o rural con la reducción de la vulnerabilidad en el menor tiempo posible dentro de un umbral aceptable que facilite la estabilidad. Asimismo, el proceso de adaptación no está acorde a las condiciones de disponibilidad de agua sino, se sigue extrayendo más de lo debido por la constante demanda de los usuarios y aunque está dentro del umbral permitido para mantener el agua con resiliencia alta no se toman las medidas para cuidar las zonas de recarga.

El umbral del agua, se encuentran dentro de los límites permisibles, lo cual la localidad de San Pedro Tultepec es resiliente. Por la cantidad de agua que disponen a nivel de cuenca, se ubica en el 2015 entre los 1000 y $1700 \mathrm{~m}^{3}$ /hab/año. Si bien la cantidad de agua media per cápita por habitante es suficiente para el desarrollo cotidiano de los habitantes, cabe destacar que el agua con que se cuenta no reúne la calidad ideal para consumo humano, pese a ello, la localidad en estos momentos dispone el recurso suficiente para satisfacer la demanda de la población. Dicho escenario en el mediano plazo cambiará de manera radical, principalmente por presentar un incremento urbano acelerado que contrasta con la reducción del recurso en cada año.

Aunque San Pedro Tultepec es resiliente, es vulnerable, principalmente por no poner en marcha acciones que reduzcan la contaminación de las aguas de los mantos acuíferos, la falta de tratamiento a las descargas realizadas en la Ciénega y de medidas de conservación o recuperación de las fuentes de abastecimiento que permita reducir la vulnerabilidad y aumentar la seguridad de la ciudad al conservar este recurso con la calidad para ser parte del consumo humano.

Con respecto a la transformación urbana, las medidas tomadas por parte de los comités autónomos de agua potable, están plasmadas principalmente en acuerdos dirigidas al mantenimiento y conservación de la infraestructura que distribuye el agua, en la parte ambiental se han tomado medidas de conservación de los mantos acuíferos y de las Ciénega 
Chimaliapan. Sin embargo, las medidas realizadas no han tenido el efecto de conservar el agua ni restringir el crecimiento urbano.

Las normas y estrategias de planeación excluyen a los comités de agua que no son reconocidos formalmente para controlar y distribuir el agua, sin embargo realizan actividades de administración y distribución. Asimismo, La ONU, (2002) en el apartado de Derechos Económicos, Sociales y Culturales considera el agua como un bien social y cultural y no económico, en el cual los comités es posible reconocerse bajo este derecho.

Los comités de agua son independientes y se mantienen en las comunidades que tiene Lerma por los acuerdos que se establecen entre los usuarios, tradicionalmente han mantenido el control del agua y la dotación del servicio del agua en las comunidades principalmente rurales que han organizado por secciones o barrios y cada uno de ellos cuenta con su propio comité, encargado de controlar el agua.

El comité de San Pedro Tultepec presenta desventajas para transformarse, esto se debe a la falta de mantenimiento de la red de distribución y de los pozos que tienen mayor demanda de agua lo que afecta a los pobladores que coordina cada comité. También la débil coordinación y comunicación entre normas ha evitado que se refleje en las acciones locales, además de la falta de control del crecimiento urbano y protección del ambiente merman la capacidad de transformación, ya que existe los ejidatarios que influyen indirectamente en la toma de decisiones para conservar la Ciénegas de Chimaliapan.

La comunicación entre los administradores del agua es parcial, ya que no se promueve la coordinación y colaboración entre comités y ejidatarios de San Pedro Tultepec así como del municipio de Lerma que controla el agua. Entre ellos existe el usuario externo, es el caso de la Ciudad de México que solo administra el agua para la ciudad y no involucra a los locales para mantener el recurso, además de los ejidatarios del área de las Ciénegas que funge como zona de recarga.

\section{CONCLUSIONES}

La adaptación de la localidad de San Pedro Tultepec está relacionada con las acciones de respuesta a situaciones de vulnerabilidad que desarrollan los comités. Entre las medidas que se realizan están la construcción de más pozos a más profundidad, los pozos artesanales en las viviendas y otras fuentes de abastecimiento como el servicio de pipas de agua potable y cisternas, aunque el comité de agua fomenta la resiliencia urbana sólo se observa de forma 
coyuntural, no presentan estrategias y políticas de inversión a futuro para el manejo del agua. Cabe destacar que dichas medidas a mediano plazo responderán de manera menos eficiente, por la reducción de los cuerpos de agua y principalmente por los niveles de contaminación que presentan los mantos acuíferos.

La resiliencia de las comunidades rurales, donde la escasez es recurrente como en San Pedro Tultepec, obliga a los habitantes a llevar acciones que satisfagan su demanda del recurso agua los obliga a tomar medidas de adaptación rápidamente. Entre los hallazgos: OPDAPAS y la iniciativa privada administran el agua sólo a ciertos sectores de la población, derivado de esto, cuando falla el servicio en alguno de ellos no afecta a todo el municipio, por lo que el comité de agua de San Pedro Tultepec no se ve afectado. La transformación de los comités está orientada a políticas y acuerdos que se realizan parcialmente sin ninguna vigilancia y visión a futuro.

La dinámica entre la demanda y la disponibilidad del agua de San Pedro Tultepec los ubica en la fase de respuesta con oscilaciones que buscan la estabilidad para adaptarse y transformarse, sin embargo, aún no presentan aspectos críticos que les permita cruzar el umbral, por lo que están ubicados en un intervalo de vulnerabilidad de manera estable que les ha permitido mantenerse frente a la escasez. El agua que les falta en tiempo de estiaje es abastecida por pipas y pozos artesanales.

La nula relación estructural entre el comité, OPDAPAS y la iniciativa privada ha permitido que la autonomía de cada uno plantee respuestas aisladas y particulares no generalizadas, por ello no afectan al sistema del comité del agua, por lo tanto, la resiliencia urbana también presenta un margen de vulnerabilidad aceptable que favorece la adaptación y transformación en la estructura del comité para el manejo y control del agua.

\section{RECONOCIMIENTO}

A beca CONACYT por haber hecho posible la realización de la presente investigación como becario con numero 411751 durante el programa de Posgrado del Doctorado en Urbanismo de la Facultad de Planeación Urbana y Regional de la Universidad Autónoma del Estado de México. 


\section{REFERENCIAS}

BÉNÉ, Christophe et al. Exploring the Potential and Limits of the Resilience Agenda in Rapidly Urbanising Contexts. Disponível em: <http://www.ids.ac.uk/publication/exploring-the-potentialand-limits-of-the-resilience-agenda-in-rapidly-urbanising-contexts>. Acesso em: 16 out. 2016.

CAMACHO, Gloria. El problema agrario en el municipio de Lerma, Estado de México: el caso de la laguna de Lerma, 1880-1930. Boletín del Archivo Histórico del Agua v. 12, n. 36, p. 7-21 , 2007.

CARDONA, Omar D. Gestión integral de riesgos de desastres Colombia, Manizales, Curso de Maestría en Medio Ambiene y Desarrollo . [S.I.]: Instituto de Estudios Ambientales., 2005

FOLKE, Carl et al. Ecology and Society: Resilience Thinking: Integrating Resilience, Adaptability and Transformability. 2010.2 Disponível em: <http://www.ecologyandsociety.org/vol15/iss4/art20/>. Acesso em: 18 set. 2016.

GEM. Ayuntamiento de Lerma » Historia . [S.I: s.n.]. Disponível em: <http://www.lerma.gob.mx/municipio/historia/>. Acesso em: 29 dez. 2017. , 2016

GEM. Plan de Desarrollo Municipal de Lerma 2013 Gaceta Municipal. Periódico oficial de gobierno municipal de Lerma, Estado de México. Nueva época 07, miércoles 10 de Abril de 2013. Disponível em: <http://www.lerma.gob.mx/wp-content/uploads/docspages/plan_desarrollo_municipal_lerma.pdf>. Acesso em: 14 jun. 2016.

GEM. Prontuario de información geográfica municipal de los Estados Unidos Mexicanos: Lerma . [S.I: S.n.]. Disponível em: <http://www3.inegi.org.mx/contenidos/app/mexicocifras/datos_geograficos/15/15051.pdf>. , 2009

GIBBERD, Jeremy. Assessing and Intervening: Urban Resilience Indicators. Disponível em: <http://www.academia.edu/4400216/Assessing_and_Intervening_Urban_Resilience_Indicators >. Acesso em: 8 out. 2016. 
HOLLING, C. S. Resilience and Stability of Ecological Systems. Annual Review of Ecology and Systematics v. 4, n. 1, p. 1-23, nov. 1973.

INEGI. Mapas. Disponível em: <http://www.beta.inegi.org.mx/app/mapas/>. Acesso em: 3 jan. 2018.

LIAO, Kuei-Hsien. A Theory on Urban Resilience to Floods--A Basis for Alternative Planning Practices. Ecology and Society v. 17, n. 4 , 2012. Disponível em: <http://www.ecologyandsociety.org/vol17/iss4/art48/>. Acesso em: 23 jun. 2016.

LÓPEZ-VILLAMAR, Sara M.; MARTínEZ-SALDAÑA, Tomás; PALERM-VIQUEIRA, Jacinta. Las comunidades en la administración de sistemas de agua potable: región de los volcanes, Estado de México. Agricultura, sociedad y desarrollo v. 10, n. 1, p. 39-58, mar. 2013.

MACLUNE, K; TYLER, K; ALLAN, C. Introduction to a New Approach to Urban Resilience Red Cross/Red Crescent National Societies. Disponível em: <http://i-s-e-t.org/resources/majorprogram-reports/approach-urban-resilience.html>. Acesso em: 29 dez. 2017.

MCPHEARSON, Timon et al. Scientists Must Have a Say in the Future of Cities - IIHS Knowledge Gateway . [S.I: s.n.]. Disponível em: <http://iihs.co.in/knowledge-gateway/scientists-must-havea-say-in-the-future-of-cities/>. Acesso em: 17 mar. 2017. , 2016

OCHOA, Carlos. Muestreo no probabilístico: muestreo por bola de nieve. Disponível em: <https://www.netquest.com>. Acesso em: 14 fev. 2018.

ONU. Cuestiones Sistantivas que se plantean en la aplicación del pacto internacional de derechos económicos, sociales y culturales: Observación general No 15 (2002) Consejo $\begin{array}{llll}\text { Económico } & \text { y } & \text { Social. } & \text { Disponível }\end{array}$ <http://www.acnur.org/t3/fileadmin/Documentos/BDL/2012/8789.pdf?view=1>. Acesso em: 29 dez. 2017. 
ONU-HABITAT. Approaches to building resilience | cityresilience.org http://cityresilience.org/sites/all/docs/Brochures/SP_City_Engagement_Steps.pdf. Disponível em: <http://cityresilience.org/approaches-building-resilience>. Acesso em: 30 dez. 2017.

ROCKSTRÖM, Johan et al. Water Resilience for Human Prosperity. [S.I.]: Cambridge University Press, 2014. 315 p. 978-1-107-02419-9.

SENGUPTA, Norimitsu Onishi y Somini. Esta ciudad sudafricana está cerca de su "día cero" por la escasez del agua. The New York Times 31 jan. 2018 Disponível em: <https://www.nytimes.com/es/2018/01/31/ciudad-del-cabo-sudafrica-sequia-agua/>. Acesso em: 20 fev. 2018Noticias.

SHARIFI, A.; YAMAGATA, Y. Major principles and criteria for development of an urban resilience assessment index. In: 2014 INTERNATIONAL CONFERENCE AND UTILITY EXHIBITION ON GREEN ENERGY FOR SUSTAINABLE DEVELOPMENT (ICUE), mar. 2014, [S.I: s.n.], mar. 2014. p.1-5.

TKF. Bounce Forward: Urban Resilience in the Era of Climate Change. Disponível em: <http://kresge.org/library/bounce-forward-urban-resilience-era-climate-change>. Acesso em: 25 set. 2017.

VANGUARDIA. Venecia ya no se hunde, ahora se queda sin agua. Disponível em: <http://www.lavanguardia.com/internacional/20180203/44497555688/venecia-canales-sinagua.html>. Acesso em: 20 fev. 2018.

Trabalho enviado em 27 de abril de 2018

Aceito em 19 de agosto de 2018 